\title{
COLOUR DOPPLER SONOGRAPHY: A NEW INVESTIGATIVE MODALITY FOR INTRAOCULAR SPACE- OCCUPYING LESIONS
}

\author{
ARUN C. GULANI ${ }^{1}$, H. MORPARIA ${ }^{2}$, S. S. BHATTI ${ }^{1}$ and R. P. JEHANGIR ${ }^{1}$ \\ Bombay, India
}

\begin{abstract}
SUMMARY
We report our experience with colour Doppler sonography (CDS) in 20 normal subjects and 25 patients with intraocular space-occupying lesions. The technique provided an in vivo cross-section of the vascular topography of space-occupying lesions in real time. Colour coding and spectral analysis helped to determine the direction and pattern of blood flow. CDS was found to be a useful diagnostic adjuvant, a guide to the treatment protocol, and helped in monitoring the progress of the disease. In conditions of the eye where unclear media hindered a clinical evaluation, CDS sonography was a safe, easily reproducible and non-invasive investigative tool. With further technical advances in waveform analysis and quantitative assessment, CDS holds promise as an investigative modality of choice in the future.
\end{abstract}

Colour Doppler sonography is currently used in the fields of cardiology, ${ }^{\prime}$ cerebrovascular disease, ${ }^{2}$ genito-urinary vascular studies, peripheral arterial and venous disorders and neonatal intracranial vascular studies. ${ }^{3}$ There are limited reports in literature of its application to the study of the eye and orbit. We report our experience and results of the use of colour Doppler sonography in 45 cases.

\section{PATIENTS AND METHODS}

Colour Doppler sonography was performed using a 7.5 $\mathrm{MHz}$ linear array transducer (Acuson $128 \mathrm{XP}$ ). The eyes were examined with the patient supine and eyelids closed. The transducer was applied to the closed eyelids using a coupling gel.

This system enables detection of amplitude, phase and frequency shift, revealing a real-time grey-scale/colour flow image. Antegrade (i.e. towards the transducer) arterial flow was selected to be shown in red and venous

From: 'Ophthalmology Department, King Edward VII Memorial Hospital, Parel, Bombay, India; ${ }^{2}$ N. M. Medical Centre, Bombay, India.

Correspondence to: Dr Arun C. Gulani, 'Anita' Apts., C/486 Pali Road, Bandra (W), Bombay 400 050, India. flow (away from the transducer) in blue. Spectral analysis also helped to distinguish between pulsatile arterial flow and continuous or minimally pulsatile venous flow.

The eyes of 20 healthy subjects (the control group) were examined to establish the sites of evaluation and to provide a comparison with the abnormal findings seen in the affected eyes of the patients. Details of the cases studied are shown in Table I. Most of these pathologies were diagnosed by clinical examination, direct and indirect ophthalmoscopy, fluorescein angiography, and conventional B-scan ultrasonography where applicable.

\section{RESULTS}

The control group subjects were healthy individuals ranging in age from 28 to 50 years (mean 39 years). There were 12 men and 8 women.

\section{Choroidal Malignant Melanoma}

Four patients with unilateral malignant melanoma (mean age 42 years) were subjected to colour Doppler imaging. Doppler signals were elicited in all the melanomas and tumour vessels identified by colour imaging (Fig. 1). Irrespective of media clarity, colour Doppler provided an in vivo cross-section of the vascular topography of the tumour in real time. Maximum systolic velocity from the tumour vessels ranged between 17 and $23 \mathrm{~cm} / \mathrm{s}$.

\section{Choroidal Tuberculoma}

Two patients with choroidal tuberculoma (mean age 32.5

Table I. Cases studied

\begin{tabular}{lccc}
\hline & \multicolumn{3}{c}{ No. of cases } \\
\cline { 2 - 4 } Type of disease & Unilateral & Bilateral & Total \\
\hline Choroidal melanoma & 4 & - & 4 \\
Choroidal tuberculoma & 2 & - & 2 \\
Retinoblastoma & 8 & 2 & 10 \\
Persistent hyperplastic primary & 3 & - & 3 \\
vitreous & 5 & 1 & 6 \\
Angiomatosis retinae & & & 25 \\
\hline
\end{tabular}

Eye (1994) 8, 307-310 C 1994 Royal College of Ophthalmologists 
years) were studied. Ophthalmoscopic examination revealed a choroidal mass with exudative retinal detachment around it. Fluorescein angiography showed blocked fluorescence in the affected area and no tumour vessels within the mass. Colour Doppler confirmed the absence of intrinsic vasculature within the mass and revealed vessels coursing over the detached retina in the echo-free vitreous cavity (Fig. 2). Investigations for oculotoxoplasmosis and syphilis were negative. A total body scan failed to reveal any occult primary malignancy elsewhere. A chest radio-

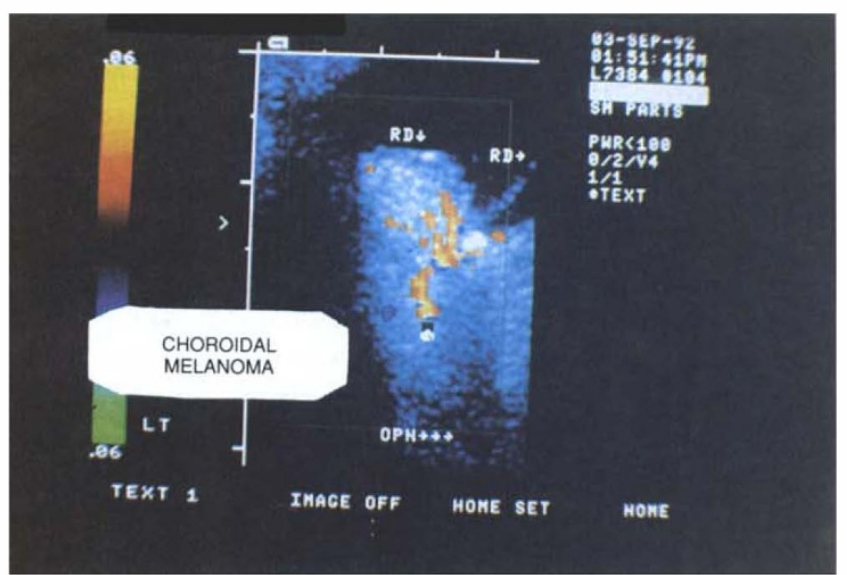

Fig. 1

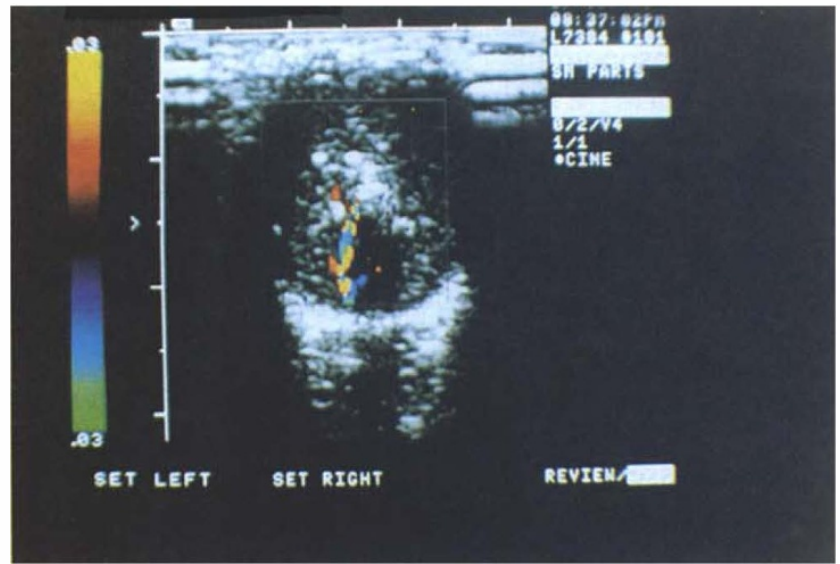

Fig. 3

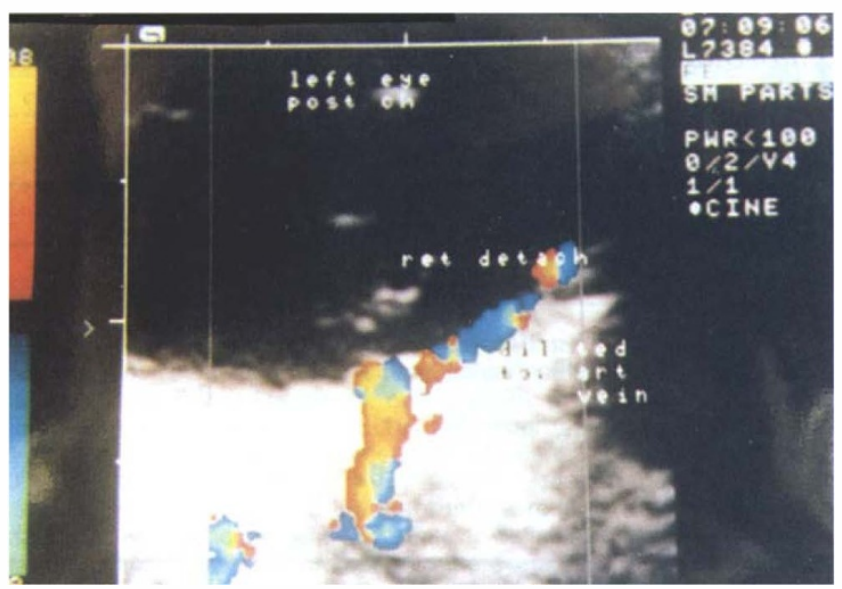

Fig. 5 graph showed evidence of inactive pulmonary tuberculosis. A positive Mantoux skin test for tuberculosis was elicited. The patient improved on anti-tuberculous chemotherapy.

\section{Retinoblastoma}

Ten patients with retinoblastoma (mean age 1.5 years) were studied. Eight cases were unilateral, of which 7 presented with clear media and 1 as a complicated cataract. Two patients had bilateral involvement. Diagnosis was

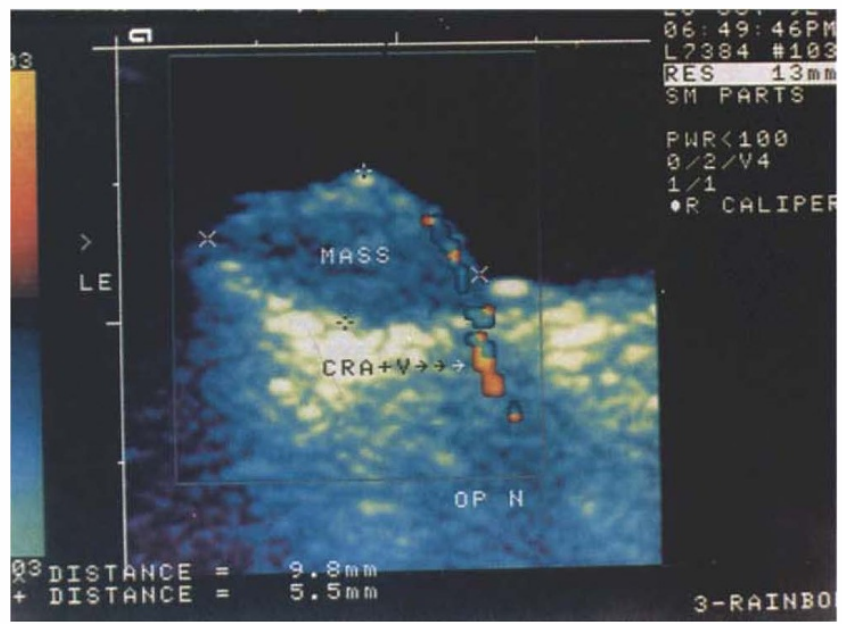

Fig. 2

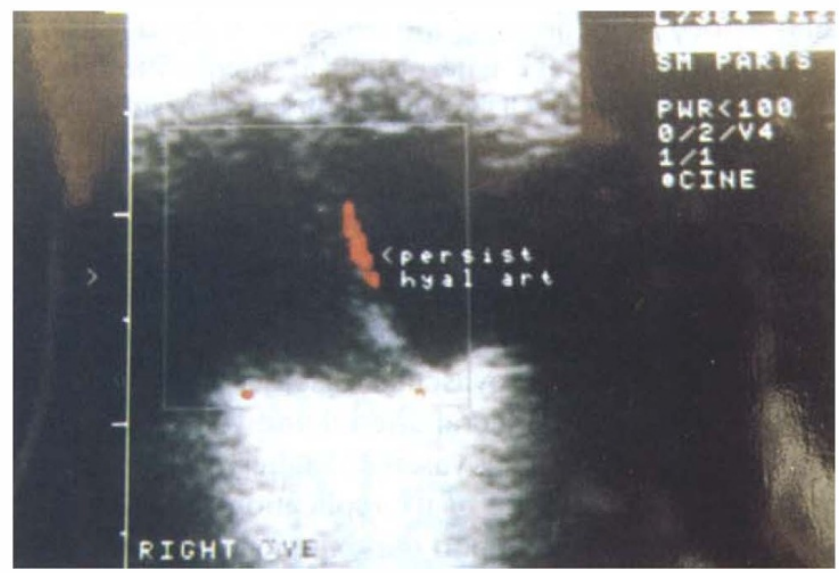

Fig. 4

Fig. 1. Feeder vessel seen within a choroidal melanoma.

Fig. 2. Absence of feeder vessel within a choroidal tuberculoma. Retinal vessels are seen on the detached retina in the echo-fice vitreous.

Fig. 3. Feeder vessel supplying a retinoblastoma. Calcification is evident within the tumour.

Fig. 4. Patent vessel present in the fibrous band extending from the disc to the retrolental area in a case of persistent hyperplastic primary vitreous.

Fig. 5. Dilated, tortuous, intertwined feeder vessels coursing from the optic disc towards the angioma in a case of angiomatosis retinae. 
made on the basis of age at presentation, leucocoria and fundus examination. Conventional B-scan sonography showed the presence of an intraocular mass with evidence of calcification within it. Colour Doppler revealed typical tumour vascularity in all cases (Fig. 3). These vessels showed high flow and a maximum velocity of 18$19 \mathrm{~cm} / \mathrm{s}$. The unilateral cases underwent enucleation and histopathological confirmation. Two of the patients with bilateral involvement underwent enucleation of the worse eye and cryotherapy for the remaining eye. These eyes were followed up on colour Doppler, which showed decreased vascularity and gradual regression that was correlated ophthalmoscopically.

\section{Persistent Hyperplastic Primary Vitreous}

Three patients with persistent hyperplastic primary vitreous (mean age 10 months) were studied. Patients presented with unilateral leucocoria and microphthalmos. B-scan sonography showed the presence of a band coursing from the optic disc to the peripheral retina and lens. Colour Doppler revealed the presence of a patent hyaloid vessel within the band in all our cases (Fig. 4), thus confirming the diagnosis and overruling the other relevant causes of leucocoria at this age.

\section{Von Hippel's Disease (Angiomatosis Retinae)}

Six cases of Von Hippel's disease were evaluated. Five of these were unilateral and 1 bilateral. Fundus examination of the affected eye showed the presence of an angiomatous mass in the mid-periphery of the retina and the presence of dilated, tortuous feeder vessels coursing from the optic disc to the angioma. Ultrasonography demonstrated the mass with minimal surrounding retinal detachment. Colour Doppler imaging showed a pair of dilated, tortuous, intertwined blood vessels arising from the optic disc and coursing to the angioma (Fig. 5). The flow in these two vessels was in opposite directions as depicted by the colour mode. Peak velocity in the artery was 19$22 \mathrm{~cm} / \mathrm{s}$ (mean $20.5 \mathrm{~cm} / \mathrm{s})$. This colour Doppler picture was consistent in all our 6 cases. We stress the importance of these findings as highly suggestive of angiomatosis retinae.

\section{DISCUSSION}

We initially studied a control group to familiarise ourselves with the normal vascularity and sono-anatomy of the eye.

Conservative therapy of malignant melanoma of the choroid necessitates improved methods of assessing these tumours before and after treatment. Though fluorescein angiography is helpful it requires the presence of clear ocular media. A-scan ultrasonography cannot elucidate the vascular topography or identify low-flow vascular nests in treated tumours. The vasculature of the malignant melanoma plays an important role in its conservative management. Better-perfused tissue is more radiosensitive than poorly perfused tissue. ${ }^{+}$The fact that colour Doppler is independent of media clarity and is able to define the vascularity thus holds significance in the diagnosis and management of these tumours. In monitoring malignant melanomas following radiotherapy the presence of vascular channels identified on colour Doppler may indicate a lingering tumour viability, and thus affect prognosis.

The ability to rule out a malignant mass in our case of choroidal tuberculoma prevented the patient from undergoing further expensive and hazardous investigations and morbid surgery.

The importance of Doppler sonography in diagnosing and monitoring retinoblastoma, especially in the presence of unclear optical media, needs to be stressed, as not all cases show the presence of calcification on conventional B-scan ultrasonography.

The presence of a patent hyaloid vessel within the fibrous band extending from the optic disc to the lens helps in confirming the diagnosis of persistent hyperplastic primary vitreous; also the presence of a patent vessel will affect the planning of surgery.

The consistency with which we were able to picture the cases of angiomatosis retinae would be of value in diagnosing this condition in cases where visualisation of the ocular fundus is not possible ophthalmoscopically due to opaque media (which may be a complication of the disease). Colour Doppler sonography could be added to the existing protocol for evaluating affected patients and relatives at risk of having the abnormal gene. ${ }^{6}$

Colour Doppler combines anatomical and velocity data, displaying a picture which is easily comprehended. This has revolutionised non-invasive vascular imaging techniques and for the first time ocular and orbital vasculature has been subjected to imaging in an informative and reproducible manner. The various advantages of colour Doppler sonography compared with other diagnostic modalities are that it: (1) is safe, and non-invasive, (2) does not require contrast material, (3) is relatively inexpensive, (4) is quick and repeatable, (5) has consistent reproducibility, (6) is easy to carry out and comprehend, and (7) provides specific topographic information regarding intraocular space-occupying lesions along with their vasculature in a dynamic, real-time setting.

Colour Doppler sonography has recently been used in the field of ophthalmology in the investigation of caroticocavernous fistula, ${ }^{7}$ carotid artery disease, ${ }^{8}$ orbital varix ${ }^{9}$ superior ophthalmic vein thrombosis ${ }^{10}$ and central retinal vessel evaluation." We now stress the importance of this new technique as a useful adjunct to the existing battery of investigative modalities in the diagnosis and management of intraocular space-occupying lesions.

We thank the Seth G. S. Medical College \& KEM Hospital, Diamond Jubilee Society Trust for financial aid towards this project. We also thank Dr N. Shah, Director of the N. M. Medical Centre, Bombay.

Key words: Central retinal vessels. Colour Doppler sonography, Fluorescein angiography. Frequency, Grey-scale imaging, L.eucocoria. Opaque/unclear area, Spectral analysis.

\section{REFERENCES}

1. Duncan WJ. Color Doppler in clinical cardiology. Philadelphia: Saunders, 1988:1-5. 
2. Grant EG. Advances in vascular imaging with ultrasound. Ann Intern Med 1990;112:203-6.

3. Mitchell DG, et al. Neonatal brain: colour Doppler imaging. I. Technique and vascular anatomy. Radiology 1988;167: 303-6.

4. Bloomer WD, Hellman S. Normal tissue responses to radiation therapy. N Engl J Med 1975;293:80-3.

5. Ossoinig K. Standardised ophthalmic echography of the eye, orbit and periorbital region: a comprehensive slide set and study guide. 3rd ed. lowa City: Goodfellow, 1985:37-8.

6. Moore AJ. Ophthalmological screening of VHL disease. Eye 1991:5:723-8.

7. Flaharty PM, et al. Colour Doppler imaging, a new non- invasive technique to diagnose and monitor carotid cavernous sinus fistulas. Arch Ophthalmol 1991;109:522-6.

8. Lieb WE, et al. Colour Doppler imaging provides accurate assessment of orbital blood flow in occlusive carotid artery disease. Ophthalmology 1991;98:548-52.

9. Lieb WE, et al. Colour Doppler imaging in the demonstration of an orbital varix. Br J Ophthalmol 1990;74:305-8.

10. Flaharty PM, et al. Colour Doppler imaging of superior ophthalmic vein thrombosis. Arch Ophthalmol 1991;109: 582-3.

11. Guthoff RF, et al. Doppler ultrasonography of the ophthalmic and central retinal vessels. Arch Ophthalmol 1991;109: 532-6. 